\title{
Agon, composición electroacústica de Horacio Vaggione: una propuesta metodológica de análisis
}

\author{
Agon, an Electroacoustic Composition by Horacio Vaggione: \\ A Methodological Analysis Proposal
}

Agon, composição eletroacústica de Horacio Vaggione: uma proposta de análise metodológica

Luis Antonio Santillán Varela
Universidad Autónoma del Estado de Hidalgo, México
luissv@uaeh.edu.mx
https://orcid.org/0000-0003-3745-5685
Fabrizio Ammetto
Universidad de Guanajuato, México
fammetto@ugto.mx
https://orcid.org/0000-0002-2694-6393
Alejandra Béjar Bartolo
Universidad de Guanajuato, México
a.bejarbartolo@ugto.mx
https://orcid.org/0000-0002-2185-9768

\section{Resumen}

En este artículo se presenta la obra electroacústica Agon (1998) del compositor argentino Horacio Vaggione (1943-), relacionada con dos composiciones anteriores del mismo autor: Schall (1995) y Nodal (1997). Se ofrece una propuesta metodológica de análisis musical basada en los conceptos y reflexiones del propio compositor (tales como micromontaje, mixidad, procesamiento, etc.), con el objetivo de apreciar todas sus dimensiones y componentes, así como sus técnicas de realización y estructuración, todo lo anterior con el fin último de proponer una mejor recepción y apreciación de esta obra. 
Revista Iberoamericana

de las Ciencias Sociales y

Humanísticas

ISSN: $2395-7972$

Palabras clave: análisis espectral, análisis estructural, análisis musical, música electroacústica, sonograma, técnica de composición.

\begin{abstract}
The article presents the electroacoustic work Agon (1998) by the Argentine composer Horacio Vaggione (1943-), relating to two previous compositions by the same author: Schall (1995) and Nodal (1997). A methodological proposal for musical analysis based on the concepts and reflections of the composer himself (such as micro-assembly, mixidad, processing, etc.) is offered, in order to appreciate all its dimensions and components, as well as its techniques of realization and structuring: all the above to propose a better reception and appreciation of this work.
\end{abstract}

Keywords: spectral analysis, structural analysis, musical analysis, electroacoustic music, sonogram, composition technique.

\title{
Resumo
}

Este artigo apresenta a obra eletroacústica Agon (1998) do compositor argentino Horacio Vaggione (1943-), relacionada a duas composições anteriores do mesmo autor: Schall (1995) e Nodal (1997). Uma proposta metodológica de análise musical é oferecida com base nos conceitos e reflexões do próprio compositor (como micromontagem, mixidade, processamento, etc.), a fim de valorizar todas as suas dimensões e componentes, bem como sua produção e estruturação técnicas, todas as anteriores com o objetivo final de propor uma melhor recepção e apreciação deste trabalho.

Palavras-chave: análise espectral, análise estrutural, análise musical, música eletroacústica, sonograma, técnica de composição.

Fecha Recepción: Julio 2020

Fecha Aceptación: Diciembre 2020 
Revista Iberoamericana

de las Ciencias Sociales y

Humanísticas

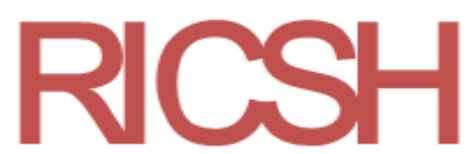

ISSN: $2395-7972$

\section{Introducción}

La importancia de contar con un método que permita realizar un análisis adecuado de obras musicales electroacústicas, digitales y experimentales es cada día más grande debido a la cada vez mayor cantidad de música que se crea en la nueva era de la tecnología digital: esta propuesta pretende brindar herramientas necesarias a los nuevos creadores de obras musicales asistidas por diversos medios. El hecho de poder disfrutar y apreciar al máximo una obra musical tiene que ver directamente con el entendimiento de la estructura, la belleza estética y muchos otros parámetros. Para un conocimiento adecuado del proceso creativo de un compositor, es necesario hacer referencia a determinados factores que van más allá de la cuestión formal de su obra, con el fin de entenderla y apreciarla en los niveles más elevados.

En este artículo mostramos una exploración del método propuesto a través del análisis de la obra maestra Agon de Horacio Vaggione, ${ }^{1}$ compositor argentino que se ha dedicado al género de la música electroacústica desde el siglo XX (Dal Farra, 2004). El método de análisis que proponemos está basado en conceptos clásicos de análisis musical, estructural, filosófico y estético, combinados con nuevas tendencias de inclusión tecnológica. El objetivo general es brindar al lector una propuesta de análisis musical aplicado a la obra en cuestión para su mejor comprensión y apreciación; dicho objetivo se deriva de la hipótesis que da pie a la necesidad de contar con un método que permita analizar obras musicales electroacústicas y de arte sonoro digital de manera eficaz y útil.

\section{Fundamentación}

Horacio Vaggione, nacido en Córdoba (Argentina), dio luz a una obra llamada Agon; la concibió en París, en 1998, cuando era director del Centre de Recherches Informatique et Création Musicale de París. En Agon, Vaggione refine los procesos y materiales anteriormente escuchados en otra composición que había realizado previamente en el año 1997 — según palabras del propio autor — a la cual llamó Nodal, y esta a su vez se basó en otra obra previa llamada Schall (Vaggione, 1995). Las tres obras son realizadas por los nuevos sistemas de computadora personal que ya se ofrecían para esta época; de este modo, se representa con ellas una especie de suite que se puede pensar como una sola obra evolutiva dividida en tres partes. Este hecho cobra vital importancia ya que a esas alturas del proceso creativo el compositor había pasado anteriormente por

\footnotetext{
${ }^{1}$ La composición está disponible en YouTube: https://www.youtube.com/watch?v=Y152HiUuCtQ.
} 
Revista Iberoamericana

de las Ciencias Sociales y

Humanísticas

ISSN: $2395-7972$

varias etapas compositivas que fueron sucediéndose como una evolución basada en dos principios básicos: por un lado, dependía específicamente de los medios tecnológicos que se tenían al alcance en determinados momentos de la etapa de producción del autor, como el uso de computadoras de código binario, o bien lenguajes de programación que, si bien en su momento fueron innovaciones maravillosas, poco a poco se fueron viendo consumidos por las nuevas creaciones y evoluciones; por otro lado, tenemos la parte de la evolución en el sentido de madurez creativa que va cerrando el círculo en el proceso estético filosófico que plantea cada compositor a lo largo de toda su obra. Sin embargo, por razones metodológicas propuestas por diferentes estudiosos en la materia, debemos dejar en claro que este enfoque de los cambios tecnológicos y el de la madurez del propio autor son cuestiones que de ninguna manera deben formar parte del análisis de una obra electroacústica. Al ser una obra creada en el año 1998, Agon presenta diferencias considerables en ambos sentidos respecto a las obras previas a Schall; pero al mismo tiempo, esto es parte fundamental de su ideología estético-creativa, ya que el autor sigue presentando recursos basados en sus propios campos de especialización, como el de granulación o escultura morfológica (Vaggione, 2001), técnicas que nunca fueron abandonadas por el compositor a pesar de la evolución tecnológica a la cual fueron sometidas sus posibilidades creativas.

\section{Estado del arte}

De lo antes citado, también partimos de la importancia que tiene el análisis de la obra, más allá de saber cómo está estructurada o sistematizada, para encontrar en esto un porqué en el sentido de coherencia del producto como parte del proceso creativo del autor. Debemos mencionar los estudios previos a los cuales ha sido expuesta la obra en cuestión: encontramos como fuente valiosa de información el previo análisis que hace Curtis Roads como parte de una investigación que realiza a la obra de Vaggione, tales como el artículo valioso The Art of Articulation: The Electroacoustic Music of Horacio Vaggione (Roads, 2005). Curtis Roads tuvo la oportunidad en su momento de corroborar cierta información de manera verbal-presencial con el compositor argentino, lo cual nos aclara el panorama en algunos sentidos: por ejemplo, el saber que se utilizaron instrumentos reales de percusión para la composición (Roads, 2005). 


\section{Surge una nueva dirección}

La bien llamada nueva dirección, que hace referencia principalmente a la nueva tendencia composicional de Vaggione, basada en los avances y cambios evolutivos en el aspecto tecnológico, se vio cristalizada en su composición electroacústica del año 1995 , Schall, y, consecuentemente, en el resto de las obras que le sucedieron, incluyendo Nodal (1997) y, por supuesto, la obra que representa nuestro objeto central de estudio, Agon (1998). Con Nodal el compositor reelaboró los materiales utilizados en Schall: por ejemplo, los cambios del timbre, del fondo y de la textura en la tercera parte, en donde la frecuencia es alta, cuyo sonido es análogo al de la lluvia sobre un techo delgado. Su densidad construye poco a poco nuevas explosiones y resonancias. El fondo tiene una textura que aparece por primera vez en el minuto 11:35 y desaparece hasta el minuto 12:09. La textura de cierre (una baja frecuencia retumbante) llega a la conclusión como lo hace también en la obra Agon, y esta conclusión presenta un largo de 39 segundos sin desaparecer. Dicha textura continúa — con una amplitud baja — durante varios segundos después de que el gesto final de la pieza presenta la conclusión de tres eventos de percusión como etiqueta, desde el principio hasta el final (como en Agon).

\section{Materiales y métodos}

Lo primero que vamos a tomar en cuenta para comenzar propiamente con el análisis de esta obra es el estudio que se hizo de manera general por Roads (2005) y que forma parte de una investigación acerca de toda la obra electroacústica de Vaggione. Utilizaremos la herramienta del sonograma a través del programa Sonic Visualiser (GNU), que nos presenta un gráfico bidimensional, un eje $\mathrm{X}$ (tiempo) y un eje $\mathrm{Y}$ (frecuencia), con el objetivo de visualizar la música y entender su diseño sonoro mediante el espectro de frecuencias y durante su desarrollo en el tiempo. El programa crea capas en donde podemos ver dinámicas, fraseos y relaciones de alturas; por lo tanto, podemos decir que este recurso puede ayudar, aportar información útil y servir como complemento a otras formas de análisis. Daremos un recorrido a través de la estructuración de la obra, basándonos en la teoría espectro-morfológica de Horacio Vaggione, derivada como tal de la de Pierre Schaeffer (1910-1995). Veremos cómo se va comportando la composición en su proceso evolutivo y trataremos de dar una interpretación en cuanto a la narrativa de la obra; por último, haremos una analogía con los aspectos tan trabajados por el propio compositor a lo largo de su carrera y que, sin duda, forman parte de esta composición. 


\section{Análisis de la composición}

\section{Tabla de análisis espectral}

El criterio para delimitar la obra Agon de Horacio Vaggione en distintas secciones está basado en los siguientes estatutos (evidenciados en la tabla 1), según una organización analítico estructural:

1) El material de origen aparece puro, o sin ser casi modificado o procesado en las secciones $\mathrm{A}, \mathrm{A}^{\prime} \mathrm{y} \mathrm{A}^{\prime \prime}$.

2) Las secciones $\mathrm{A}, \mathrm{A}^{\prime}$ y $\mathrm{A}^{\prime \prime}$ son considerablemente más pequeñas que la $\mathrm{B}$ y C, y guardan elementos en común.

3) Se considera al sonido seco final de la obra también como delimitador de cada sección y este aparece en los puntos mencionados, además de aparecer, claro está, como último sonido de toda la obra.

4) Se delimita e identifica el puente; este es pequeño y muy diferente a todas las otras secciones y sirve de conexión con la coda.

5) La coda conduce a un momento de cierre enfático y de fuerza progresiva que marca el punto cumbre de la obra. 
Revista Iberoamericana de las Ciencias Sociales y Humanísticas

Tabla 1. Horacio Vaggione, Agon: análisis espectral

\begin{tabular}{|c|c|c|c|c|c|c|}
\hline A & B & $\mathrm{A}^{\prime}$ & C & $\mathrm{A}^{\prime \prime}$ & Puente & Coda \\
\hline $\begin{array}{l}0.00 .000- \\
1.10 .727\end{array}$ & $\begin{array}{l}1.10 .727- \\
2.29 .118\end{array}$ & $\begin{array}{l}2.29 .118- \\
2.58 .422\end{array}$ & $\begin{array}{l}2.58 .422- \\
4.55 .682\end{array}$ & $\begin{array}{l}4.55 .682- \\
6.32 .740\end{array}$ & $\begin{array}{l}6.32 .740- \\
6.57 .850\end{array}$ & $\begin{array}{l}6.57 .850- \\
8.38 .840\end{array}$ \\
\hline $\begin{array}{l}11.517 \\
\text { Sonido de } \\
\text { clave }\end{array}$ & $\begin{array}{l}1.13 .723 \\
\text { Fuego corto }\end{array}$ & $\begin{array}{l}2.29 .118 \\
\text { Comienza } \\
\text { con la } \\
\text { campana } \\
\text { timbrada } \\
\text { dejada en la } \\
\text { sección } \\
\text { anterior }\end{array}$ & $\begin{array}{l}3.00 .256 \\
\text { Fuego } \\
\text { quemando } \\
\text { se convierte } \\
\text { en material } \\
\text { conductor } \\
\text { principal }\end{array}$ & $\begin{array}{l}4.55 .682 \\
\text { Se comienza con } \\
\text { el motivo } \\
\text { conductor de la } \\
\text { sección A, por } \\
\text { medio de la } \\
\text { aceleración y } \\
\text { ralentización; a } \\
\text { continuación, } \\
\text { utiliza todos los } \\
\text { motivos de la } \\
\text { mencionada } \\
\text { sección, pero los } \\
\text { granula y } \\
\text { combina de } \\
\text { muchas formas } \\
\text { hasta lograr con } \\
\text { esto hacer } \\
\text { aparecer el } \\
\text { sonido de la } \\
\text { campana } \\
\text { timbrada }\end{array}$ & $\begin{array}{l}6.32 .740 \\
\text { Comienza } \\
\text { el puente } \\
\text { con dos } \\
\text { clics } \\
\text { seguidos }\end{array}$ & $\begin{array}{l}6.57 .850 \\
\text { Comienza la } \\
\text { coda (se } \\
\text { enciende } \\
\text { fuego) }\end{array}$ \\
\hline $\begin{array}{l}27.028 \\
\text { Timbal } \\
\text { procesado }\end{array}$ & $\begin{array}{l}1.27 .980 \\
\text { Clave }\end{array}$ & $\begin{array}{l}\text { Se utiliza } \\
\text { una } \\
\text { combinació } \\
\text { n de } \\
\text { recursos } \\
\text { sonoros de } \\
\text { la sección A }\end{array}$ & $\begin{array}{l}3.18 .577 \\
\text { Se enciende } \\
\text { fuego }\end{array}$ & $\begin{array}{l}5.51 .259 \\
\text { Gran campana } \\
\text { forte }\end{array}$ & $\begin{array}{l}6.47 .719 \\
\text { Segunda } \\
\text { parte del } \\
\text { puente con } \\
\text { dos clics } \\
\text { seguidos }\end{array}$ & $\begin{array}{l}7.42 .470 \\
\text { Campana }\end{array}$ \\
\hline
\end{tabular}


Revista Iberoamericana de las Ciencias Sociales y Humanísticas

ISSN: 2395 - 7972

\begin{tabular}{|c|c|c|c|c|c|}
\hline $\begin{array}{l}33.901 \\
\text { Timbal } \\
\text { procesado }\end{array}$ & $\begin{array}{l}1.32 .670 \\
\text { Clave }\end{array}$ & $\begin{array}{l}2.35 .422 \\
\text { Se termina } \\
\text { la campana } \\
\text { timbrada }\end{array}$ & $\begin{array}{l}3.20 .051- \\
3.31 .127 \\
\text { Se presenta } \\
\text { una serie de } \\
\text { cuatro } \\
\text { explosiones }\end{array}$ & $\begin{array}{l}5.59 .494 \\
\text { La campana se } \\
\text { convierte en } \\
\text { campana } \\
\text { timbrada } \\
\text { durante toda la } \\
\text { sección }\end{array}$ & $\begin{array}{l}7.50 .760 \\
\text { Timbal }\end{array}$ \\
\hline $\begin{array}{l}37.848 \\
\text { Campana } \\
\text { seca }\end{array}$ & $\begin{array}{l}1.35 .364 \\
\text { Combinació } \\
\text { n de fuego, } \\
\text { timbal, } \\
\text { campana } \\
\text { seca y } \\
\text { campana } \\
\text { timbrada }\end{array}$ & $\begin{array}{l}2.37 .704 \\
\text { Efecto de } \\
\text { fuego } \\
\text { quemando }\end{array}$ & $\begin{array}{l}3.42 .122- \\
3.44 .002 \\
\text { Episodio de } \\
\text { platillos } \\
\text { secos }\end{array}$ & $\begin{array}{l}6.02 .347 \\
\text { Patinado de } \\
\text { recursos sonoros }\end{array}$ & $\begin{array}{l}8.05 .220 \\
\text { Timbal } \\
\text { procesado }\end{array}$ \\
\hline $\begin{array}{l}57.161 \\
\text { Campana } \\
\text { timbrando }\end{array}$ & $\begin{array}{l}1.46 .091- \\
1.52 .593 \\
\text { Episodio de } \\
\text { platillos } \\
\text { secos }\end{array}$ & $\begin{array}{l}2.53 .453 \\
\text { Explosión }\end{array}$ & $\begin{array}{l}4.10 .322 \\
\text { Explosión } \\
\text { que va en } \\
\text { crescendo }\end{array}$ & $\begin{array}{l}6.07 .960 \\
\text { Timbal y } \\
\text { campana } \\
\text { procesados }\end{array}$ & $\begin{array}{l}8.12 .120 \\
\text { Se } \\
\text { reenciende } \\
\text { el fuego, que } \\
\text { consume } \\
\text { todo hasta el } \\
\text { final en un } \\
\text { diminuendo }\end{array}$ \\
\hline $\begin{array}{l}1.02 .879 \\
\text { Timbal } \\
\text { procesado y } \\
\text { se apaga la } \\
\text { campana }\end{array}$ & $\begin{array}{l}\text { 1.53.708 } \\
\text { Timbal } \\
\text { procesado }\end{array}$ & & $\begin{array}{l}\text { 4.13.151 } \\
\text { Fuerte } \\
\text { explosión } \\
\text { que hace } \\
\text { aparecer a } \\
\text { la campana } \\
\text { timbrada y a } \\
\text { los platillos }\end{array}$ & $\begin{array}{l}6.15 .385 \\
\text { Pequeña } \\
\text { explosión }\end{array}$ & $\begin{array}{l}8.33 .810 \\
\text { Sonido } \\
\text { granulado } \\
\text { como cierre }\end{array}$ \\
\hline $\begin{array}{l}1.04 .969 \\
\text { Timbal } \\
\text { procesado }\end{array}$ & $\begin{array}{l}2.01 .951 \\
\text { Fuego } \\
\text { mayor en }\end{array}$ & & $\begin{array}{l}4.28 .701 \\
\text { Explosión }\end{array}$ & $\begin{array}{l}6.16 .429 \\
\text { Gran explosión }\end{array}$ & $\begin{array}{l}8.38 .840 \\
\text { Sonido seco } \\
\text { final }\end{array}$ \\
\hline
\end{tabular}


Revista Iberoamericana de las Ciencias Sociales y Humanísticas

\begin{tabular}{|c|c|c|c|}
\hline & $\begin{array}{l}\text { forte y va } \\
\text { disminuyen } \\
\text { do }\end{array}$ & & \\
\hline $\begin{array}{l}1.10 .727 \\
\text { Sonido seco } \\
\text { final }\end{array}$ & $\begin{array}{l}2.17 .020 \\
\text { Fuego } \\
\text { disminuido }\end{array}$ & $\begin{array}{l}4.34 .877 \\
\text { Explosión } \\
\text { procesada }\end{array}$ & $\begin{array}{l}6.32 .740 \\
\text { Sonido seco } \\
\text { final que parte } \\
\text { de dos clics } \\
\text { seguidos }\end{array}$ \\
\hline & $\begin{array}{l}\text { Comienza } \\
\text { una } \\
\text { granulación } \\
\text { de sonidos }\end{array}$ & $\begin{array}{l}4.37 .803 \\
\text { Explosión } \\
\text { procesada }\end{array}$ & \\
\hline & $\begin{array}{l}\text { Comienza } \\
\text { la campana } \\
\text { timbrando }\end{array}$ & $\begin{array}{l}4.40 .613 \\
\text { Proceso de } \\
\text { recursos }\end{array}$ & \\
\hline & $\begin{array}{l}2.29 .118 \\
\text { Sonido seco } \\
\text { final }\end{array}$ & $\begin{array}{l}4.43 .387 \\
\text { Enciende } \\
\text { fuego con } \\
\text { fuerte } \\
\text { explosión }\end{array}$ & \\
\hline & & $\begin{array}{l}4.55 .682 \\
\text { Sonido seco } \\
\text { final }\end{array}$ & \\
\hline
\end{tabular}

Fuente: Elaboración propia

\section{Resultados}

La obra Agon tiene una duración de 8 minutos y 43 segundos: se abre con una banda sonora de fluttering continuo entre la gama de $6 \mathrm{kHz}$ y $16 \mathrm{kHz}$. La velocidad de la modulación fluttering oscila entre $10 \mathrm{~Hz}$ y $20 \mathrm{~Hz}$. La continuidad de la banda de alta frecuencia se rompe por varias explosiones compuestas de colores diversos en ciertos momentos clave. Es como si diferentes sonidos de percusión se dejaran caer en un granulador gigante para ser acolchados instantáneamente en bits de microsonido. Un primer contacto auditivo con Agon podría mostrar simplemente un flujo continuo de nuevo material, pero un conocimiento más profundo de la obra revela un trabajo 
Revista Iberoamericana

de las Ciencias Sociales y

Humanísticas

ISSN: 2395 - 7972

compositivo de reciclaje del material sonoro de una manera sumamente eficiente: por ejemplo, en el penúltimo gesto de la obra se puede escuchar un turbio remolino en la banda de frecuencia medio-baja que ya se había escuchado en los primeros 35 segundos. En el último gesto del trabajo, un golpe triple de "tom-clic-siseo" aparece por primera vez a los 2' 59" y de nuevo a los 3' 8". Algunos de los sonidos reciclados en Agon son extrañas mutaciones de otros sonidos, mientras otros se dibujan a mano en un editor gráfico de audio y no se derivan de ninguna fuente original.

“Consideré, escuchando por primera vez, que el sonido que aparece a los 40 segundos en la pieza parece una pequeña campana de metal” (Vaggione, 2001, p. 57). Según el compositor, el origen de este sonido no era una campana, sino más bien el resultado de un procedimiento de convolución transversal de síntesis. Aparece el sonido de la "campana" por primera vez con una resonancia de $750 \mathrm{~Hz}$ y en seguida se desplaza hasta $1080 \mathrm{~Hz}$ (aproximadamente una cuarta aumentada) a los 59". Otro sonido reciclado con frecuencia es como el de un ataque de un tom-tom. Al recurrir de nueva cuenta al compositor descubrimos que esto era en realidad una forma de onda dibujada a mano. El "tom-tom" como sonido se escucha por primera vez en una ráfaga de golpes a los 34". Tanto la "campana" como el "tom-tom" reaparecen en muchos puntos dentro de Agon. Un brillante sonido como de timbal se entreteje con ellos a lo largo de toda la obra: se trata de un componente de la banda de alta frecuencia que fluye a través de la mayor parte de la pieza. Un cluster de piano, que se originó - de acuerdo con el compositor - como una mutación de un sonido de percusión, aparece por primera vez a los 2' 01". A continuación, se señala el final de una zona de reposo a los 5' 54", y esto marca un punto de inflexión del final a los 8' 10".

Es importante mencionar que Vaggione se especializó en síntesis granular, micromontaje y microsonido. La composición musical de Vaggione se ha convertido en un proceso interactivo de esculpir directamente morfologías sonoras en múltiples escalas de tiempo. Como podemos ver en Agon, la complejidad y la sutileza de esta música desafían a la descripción textual, que presenta formidables problemas del discurso. Afortunadamente, el compositor ha escrito una cantidad considerable de artículos acerca de su propio enfoque estético.

En el análisis de la obra, podemos observar el uso de la técnica de micromontaje (a pesar de la evolución tecnológica): esta técnica es un componente esencial del estilo de Vaggione. En el micromontaje, el compositor extrae partículas de archivos de sonido y las reordena en el tiempo y en el espacio. El término montaje se deriva del ámbito del 
Revista Iberoamericana

de las Ciencias Sociales y

Humanísticas

ISSN: 2395 - 7972

cine, donde alude a 'corte', ‘empalmes', 'disolución' y otras operaciones de edición de película. El término micro se refiere a la manera en que un compositor puede posicionar cada partícula del sonido precisamente en el lienzo del tiempo. Micromontaje digital se refiere a las operaciones que se ocupan de pequeñas partículas de sonido, partículas que pertenecen al dominio del microtiempo (generalmente menos de $100 \mathrm{~ms}$ ). En esto tenemos, de manera detallada, el equivalente musical del trabajo de un pintor puntillista.

En la música, desde hace mucho tiempo, el término puntillismo ha sido asociado con el estilo de "serie dispersa" de Anton Webern y sus seguidores. Irónicamente, la técnica del puntillista Georges Seurat (1859-1891), principal representante pictórico y creador de dicho movimiento, no presentaba ningún tipo de analogía con Webern. En contraposición, sus lienzos presentan una densidad de miles de pinceladas meticulosamente organizadas.

Tal vez la mejor manera de establecer una distinción entre la granulación y este micromontaje es apuntando que la primera es inevitablemente un proceso automático: el pincel del compositor se convierte en un chorro de pulverización refinado de color del sonido. Contrariamente, el micromontaje demanda un trabajo más minucioso, partícula por partícula, a la manera de un pintor puntillista, por lo tanto, todo esto exige una paciencia inusual. Cabe también señalar que, por supuesto, granulación y micromontaje son dos técnicas que pueden perfectamente entrelazarse: "Las técnicas de granulación de Vaggione comparten muchas similitudes con este micro-montaje" (Roads, 2005, p. 304).

Por medio del lenguaje de programación integrado en el sistema Carl, el compositor fue capaz de crear subclases de un objeto de sonido específico a través de transformaciones tales como estiramiento de tiempo (o pitch-shifting), en donde los sonidos transformados heredan la morfología del sonido original.

A continuación, proponemos los conceptos de análisis de la obra Agon, basados en la teoría explicada por el compositor respecto a los eventos que conforman sus composiciones en términos estructurales y que, por supuesto, forman parte de la obra en cuestión.

Tema. La presentación de las primeras figuras en la obra son el "tema": es con este tipo de figuras que Vaggione define a este concepto como tal.

- $\quad$ Mixidad. Se comienza por primera vez por la composición de figuras destinadas a los instrumentos y sus respectivas combinaciones; a continuación, el autor los registra y los analiza para poner sus singularidades morfológicas; por último, compone mediante la configuración de las transformaciones de estas figuras. 
Revista Iberoamericana

de las Ciencias Sociales y

Humanísticas

ISSN: $2395-7972$

- $\quad$ Técnica de composición. Esto significa amplificar y proyectar sus salientes morfológicos en otras regiones.

- $\quad$ Procesamiento (una técnica que actúa como un prisma). Vaggione señala que las transformaciones morfológicas que pone en práctica generan un trabajo figurativo y puede ser proyectado en las más diversas escalas temporales. En la granulación de un sonido muestreado se producen "figuraciones espaciales", gracias a la técnica de microdesincronización temporal.

- $\quad$ Figura. Componente de tema: el uso de la palabra figura se refiere (de una manera u otra) a la problemática del pensamiento temático.

Un mapa de eventos como parte de un análisis estructural, en el sentido de utilización de herramientas y su propia organización, sería el siguiente:

Tema—Mixidad—Técnica de composición—Procesamiento-Figura

- $\quad$ Análisis morfológico. Comenzaremos con la unificación de la electrónica con el instrumental; esta doble situación de espacio es la razón por la cual Vaggione no utiliza técnicas estándar (como la reverberación, el lavado, etc.): "Estas técnicas no tienen ninguna relación con la morfología inherente a un sonido y su adecuación a él" (Vaggione, 2007, p. 117).

Esto explica el uso del correlato microtemporal en la ingeniería de la señal; su uso consiste, por ejemplo, en la generación de réplicas de una forma de onda y del proceder a desincronizar sus relaciones de fase dentro de una escala microtemporal, que crea una sensación de espacio íntimamente ligado a la morfología de sonido.

Vaggione prefiere hablar de enfoque de transformación, más que de proceso, y utiliza la última palabra en un sentido más general, es decir, 'separarse del espectro'. En realidad, no existe otro tipo de sucesos importantes en el sentido de cambios morfológicos en la obra, ya que constantemente el autor vuelve a presentar los mismos recursos continuamente, sometidos a sus ya bien conocidas técnicas compositivas. 
Revista Iberoamericana

de las Ciencias Sociales y

Humanísticas

ISSN: $2395-7972$

\section{Discusión}

Agon es una obra estructurada en seis secciones que se pueden distinguir claramente a través del análisis espectral arrojado por el sonograma: se presenta un "tema" compuesto por ciertas figuras, el cual, en la tercera sección, se vuelve a presentar mezclado y transfigurado con diversas técnicas de procesamiento, entre estas secciones hay una sección B que las amalgama, y así sucesivamente se vuelve a presentar una quinta sección que es variante de la primera y tercera, una cuarta sección C las amalgama y después de una preparación muy evidente el compositor presenta una "coda", que es una combinación de mezclas de muchos elementos ya presentados a lo largo de la obra, pero tomando especial atención al punto de densidad musical que nos lleva de un ambiente más o menos calmo a un progresivo movimiento de conglomeración de discurso, esto es, la obra nos va llevando de menos a más, sin necesidad de utilizar una gran cantidad de recursos nuevos, sino con las transformaciones y variantes de los elementos básicos que son presentados desde un principio y que, de alguna manera, se van reconstituyendo, enriqueciendo, o bien evolucionando en el sentido de coherencia de discurso, lo cual la hace una obra muy bella e interesante que nos deja con una sensación de atmósfera muy fuerte y que, en un sentido lógico, podemos considerar la conclusión de una obra en tres movimientos: Schall-Nodal-Agon.

Tendríamos que hacer un análisis detallado de las otras dos obras mencionadas, pero a nuestro juicio podríamos proponer que tal vez estas tres obras juntas constituirían una amplia sonata en tres movimientos y que Agon como tal, siendo el último movimiento (por el análisis realizado), en forma de rondó con una coda: A-B-A'-C-A"-coda.

La primera y tercera sección, que contienen el evento que parece ser una campana, no son las únicas en contenerlo, la quinta sección también lo hace, pero, eso sí, desfragmentado en capas. Además, es preciso mencionar que este evento no indica el principio o final de ninguna de las secciones mencionadas, ya que, de hecho, se presenta en cada una de ellas en distintas zonas y con diferentes connotaciones estéticas y discursivas. Por lo demás, las secciones de enlace B y C podrían tomarse como B y B', si así se prefiere, ya que son muy semejantes auditivamente (aunque por razones de claridad en el análisis preferimos llamarles $B$ y $C$ ). La única sección de enlace diferente es la que nos lleva a la "coda" final, aunque hemos decidido llamarle puente, por su naturaleza progresiva y conductora.

Los alcances y limitaciones presentadas en esta investigación tienen que ver principalmente con la toma de decisiones respecto a la morfología y tamaño de cada una 
Revista Iberoamericana

de las Ciencias Sociales y

Humanísticas

ISSN: $2395-7972$

de las secciones de la obra analizada en cuestión; estas se tuvieron que ceñir a las normas tradicionales de estructuración musical y, a su vez, al análisis espectral y visual que arrojaron los distintos tipos de software utilizados para este fin. A pesar de tener que adaptar los recursos y métodos a una obra como esta, podemos mencionar que los resultados son concretos y demostrables, ya que se pueden corroborar en la tabla presentada anteriormente, con la aparición de los eventos clave registrados con exactitud en minutos y segundos.

El análisis espectral arrojado visualmente podría dar para mucho; sin embargo, esto no es tema de enfoque en nuestro trabajo. Así pues, la temática queda abierta a quienes pretendan seguir estudiando estos fenómenos a partir de nuestros fundamentos. Algunas consideraciones importantes que justifican la toma de ciertas decisiones en los resultados de nuestra nueva propuesta de análisis se presentan a continuación.

\section{Conclusiones}

Comenzaremos citando las conclusiones que el propio compositor escribe para intentar justificar las diversas razones que giran alrededor del génesis creativo de Agon:

Estoy interesado en investigar más a fondo la relación entre el medidor (como medidor del ciclo de la fuerza) y el ritmo (como un movimiento no cíclico) y esto no solo a nivel de "macro-time", por eso me centro en el nivel más microscópico al que se puede llegar con nuestras herramientas actuales (Budon, 2000, p. 20).

Después de evidenciar con la cita anterior la gran necesidad de contar con mejores herramientas tecnológicas, Vaggione también deja claro la importancia de la intervención del análisis musical, tanto estructural como estético, de las obras.

Al igual que Xenakis, también reconozco la necesidad de un equilibrio entre la composición algorítmica y la intervención directa: articular un gran flujo musical por medio de sucesos estadísticos es impensable. Pero en contraposición se depende de ciertos aspectos singulares: discontinuidades, figuras, contrastes y detalles (Vaggione, 2007, p. 119).

Una vez analizado el discurso del autor, podemos mencionar que, a través de determinadas estrategias, Vaggione tiene una habilidad desconcertante para hacer que las obras de arte parezcan un juego fácil. Los elementos están bien definidos, la estructura es clara, la técnica es obvia. Realmente es difícil entender esto por completo, pero se intuye que dentro de las obras hay algo mucho más profundo; sin embargo, la cuestión 
Revista Iberoamericana

de las Ciencias Sociales y

Humanísticas

ISSN: $2395-7972$

permanece estacionada, en la mayoría de los casos, sin buscar ningún método o herramienta que ayude a comprender otros aspectos, y en el mejor de los casos, tratar de utilizar herramientas que históricamente han servido para el mismo fin.

\section{Futuras líneas de investigación}

A través de la presente investigación se da pie a una propuesta de método analítico de obras electroacústicas y digitales, lo que dio como resultado el hallazgo de parámetros y singularidades que no podrían brindarnos otros métodos de análisis tradicionales (armónico, formal, tímbrico, etc.); de este modo se busca proporcionar herramientas actualizadas y adaptadas a los nuevos retos que para su comprensión y apreciación proponen las obras electroacústicas y de arte sonoro digital.

\section{Agradecimientos y colaboraciones}

Se agradece a Oscar Pablo Di Liscia y Pablo Cetta por sus valiosas colaboración y respaldo en el proyecto de investigación que da pie a este artículo.

Este trabajo es el fruto de un proyecto de investigación conjunto y de vinculación entre cuerpos académicos reconocidos por la Secretaría de Educación Pública (SEP): ${ }^{2}$ el cuerpo académico "Música" (UAEH-CA-61), ${ }^{3}$ al cual pertenece Luis Antonio Santillán Varela, y el cuerpo académico consolidado "Musicología" (UGTO-CA-66), ${ }^{4}$ al cual pertenecen Fabrizio Ammetto (SNI 2) y Alejandra Béjar Bartolo (SNI 1).

2 La contribución individual de cada autor en este texto es la siguiente: Luis Antonio Santillán Varela es el responsable de la impostación general del artículo, así como de los párrafos de la introducción (Fundamentación, Estado del arte, Surge una nueva dirección) y de la sección Resultados; Fabrizio Ammetto es el responsable de la redacción de los apartados Materiales y métodos y Análisis de la composición (tabla 1); Alejandra Béjar Bartolo es la responsable de la redacción del apartado Discusión y de la revisión general del artículo. Los tres autores son responsables de la elaboración en conjunto de Conclusiones y Futuras líneas de Investigación.

3 Cuya línea de generación y aplicación del conocimiento (LGAC) es "Rescate, creación y difusión de obras musicales".

4 Cuya LGAC es "Musicología histórica, sistemática y aplicada". 
Revista Iberoamericana

de las Ciencias Sociales y

Humanísticas

ISSN: 2395 - 7972

\section{Referencias}

Budon, O. (2000). Composing with Objects, Networks, and Time Scales: An Interview with Horacio Vaggione. Computer Music Journal, 24(3), 9-22. Retrieved from https://www.mitpressjournals.org/doi/abs/10.1162/014892600559399.

Dahan, K. (2005). An Associative Approach to Computer-Assisted Music Composition. Paper presented at the International Computer Music Conference 2005. Barcelona, September 5-9, 2005. Retrieved from http://hdl.handle.net/2027/spo.bbp2372.2005.175

Dal Farra, R. (1994). Some Comments about Electroacoustic Music and Life in Latin America. Leonardo Music Journal, 4, 93-94.

Dal Farra, R. (1996). A Southerner's Perspective. Computer Music Journal, 20(3), 3637.

Dal Farra, R. (2004). Some recent actions to preserve, document and disseminate electroacoustic music by Latin American composers. Paper presented at the $30^{\text {th }}$ Annual International Computer Music Conference. Coral Gables, November 1-6, 2004. Retrieved from http://hdl.handle.net/2027/spo.bbp2372.2004.002.

Di Liscia, O. P. (2004). Síntesis y procesamiento de sonido y música a través del programa Csound. Quilmes, Argentina: Universidad Nacional de Quilmes.

Garnett, G.E. (2001). The Aesthetics of Interactive Computer Music. Computer Music Journal 25(1), 21-33. Retrieved from https://www.mitpressjournals.org/doi/abs/10.1162/014892601300126089.

Laliberté, M. (2005). An analytic approach to Horacio Vaggione's Till. Contemporary Music Review, 24(4-5), 351-364. Retrieved from https://www.tandfonline.com/doi/abs/10.1080/07494460500172246.

Risset, J. C. (1971). An Introductory Catalogue of Computer Synthesized Sounds. Murray Hill, United States: Bell Telephone Laboratories.

Risset, J. C. and Mathews, M. V. (1969). Analysis of musical-instrument tones. Physics Today, 22(2), 23-30. Retrieved from https://physicstoday.scitation.org/doi/10.1063/1.3035399.

Roads, C. (2005). The art of articulation: The electroacoustic music of Horacio Vaggione. Contemporary Music Review, 24(4-5), 295-309.

Solomos, M. (2005). An introduction to Horacio Vaggione's musical and theoretical thought. Contemporary Music Review, 25(4-5), 311-325. Retrieved from https://www.tandfonline.com/doi/abs/10.1080/07494460500172139. 
Solomos, M. (2006). The Granular Connection (Xenakis, Vaggione, Di Scipio...). Paper presented at The Creative and Scientific Legacies of Iannis Xenakis International Symposium. Canada. Retrieved from https://hal.archives-ouvertes.fr/hal00770088.

Vaggione, H. (1995). Objets, représentations, opérations. Revue Ars Sonora, (2), 33-52.

Vaggione, H. (2001). Some Ontological Remarks about Music Composition Processes. Computer Music Journal, 25(1), 54-61.

Vaggione, H. (2007). Composer avec des objets, réseaux et échelles temporelles (interview de Osvaldo Budón). Dans Solomos, M. (ed.), Espaces composables: essais sur la musique et la pensée d'Horacio Vaggione (pp. 101-119). Paris, France: L'Harmattan.

Vaggione, H. (2010). Représentations musicales numériques: temporalités, objets, contextes. En Soulez, A. et Vaggione, H. (eds.), Manières de faire des sons (pp. 45-82). Paris, France: L'Harmattan. 
Revista Iberoamericana de las Ciencias Sociales y Humanísticas

ISSN: $2395-7972$

\begin{tabular}{|c|c|}
\hline Rol de Contribución & Autor (es) \\
\hline Conceptualización & $\begin{array}{l}\text { Luis Antonio Santillán Varela (principal) y Fabrizio Ammetto } \\
\text { (que apoya) }\end{array}$ \\
\hline Metodología & $\begin{array}{l}\text { Luis Antonio Santillán Varela (principal) y Fabrizio Ammetto } \\
\text { (que apoya) }\end{array}$ \\
\hline Software & Luis Antonio Santillán Varela \\
\hline Validación & Luis Antonio Santillán Varela \\
\hline Análisis Formal & $\begin{array}{l}\text { Luis Antonio Santillán Varela (igual) y Fabrizio Ammetto } \\
\text { (igual) }\end{array}$ \\
\hline Investigación & $\begin{array}{l}\text { Luis Antonio Santillán Varela (principal) y Fabrizio Ammetto } \\
\text { (que apoya) }\end{array}$ \\
\hline Recursos & $\begin{array}{l}\text { Luis Antonio Santillán Varela (principal) y Alejandra Béjar } \\
\text { Bartolo (que apoya) }\end{array}$ \\
\hline Curación de datos & Luis Antonio Santillán Varela \\
\hline $\begin{array}{l}\text { Escritura - Preparación del } \\
\text { borrador original }\end{array}$ & Luis Antonio Santillán Varela \\
\hline $\begin{array}{l}\text { Escritura - Revisión y } \\
\text { edición }\end{array}$ & Alejandra Béjar Bartolo \\
\hline Visualización & Fabrizio Ammetto \\
\hline Supervisión & Alejandra Béjar Bartolo \\
\hline Administración de Proyectos & Luis Antonio Santillán Varela \\
\hline Adquisición de fondos & Luis Antonio Santillán Varela \\
\hline
\end{tabular}

